\title{
ROCK activity regulates functional tight junction assembly during blastocyst formation in porcine parthenogenetic embryos
}

Jeongwoo Kwon, Nam-Hyung Kim, Inchul Choi

The Rho-associated coiled-coil-containing protein serine/threonine kinases 1 and 2 (ROCK1 and ROCK2) are Rho subfamily GTPase downstream effectors that regulate cell migration, intercellular adhesion, cell polarity, and cell proliferation by stimulating actin cytoskeleton reorganization. Inhibition of ROCK proteins affects specification of the trophectoderm (TE) and inner cell mass (ICM) lineages, compaction, and blastocyst cavitation. However, the molecules involved in blastocyst formation are not known. Here, we examined developmental competence and levels of adherens/tight junction ( $\mathrm{AJ} / \mathrm{TJ})$ constituent proteins, such as CXADR, OCLN, TJP1, and CDH1, as well as expression of their respective mRNAs, after treating porcine parthenogenetic four-cell embryos with Y-27632, a specific inhibitor of ROCK, at concentrations of $0,10,20,100 \mu \mathrm{M}$ for $24 \mathrm{~h}$. Following this treatment, the blastocyst development rates were $39.1,20.7,10.0$, and $0 \%$, respectively. In embryos treated with $20 \mu \mathrm{M}$ treatment, expression levels of CXADR, OCLN, TJP1, and CDH1 mRNA and protein molecules were significantly reduced $(P<0.05)$. FITC-dextran uptake assay revealed that the treatment caused an increase in TE TJ permeability. Interestingly, the majority of the four-cell and morula embryos treated with $20 \mu \mathrm{M}$ Y-27643 for $24 \mathrm{~h}$ showed defective compaction and cavitation. Taken together, our results indicate that ROCK activity may differentially affect assembly of $\mathrm{AJ} / \mathrm{TJS}$ as well as regulate expression of genes encoding junctional proteins. 
1 ROCK activity regulates functional tight junction assembly during blastocyst formation in

2 porcine parthenogenetic embryos

3

4 JeongWoo Kwon', Nam-Hyung Kim¹, Inchul Choi²

5

6 'Department of Animal Sciences, Chungbuk National University, Gaesin-dong, Cheongju,

7 Chungbuk 28864, Republic of Korea

$8{ }^{2}$ Department of Animal and Dairy Sciences, College of Agriculture and Life Sciences, Chungnam

9 National University, Daejeon, 34134, Republic of Korea.

12 Corresponding authors:

13 Nam-Hyung Kim ${ }^{1}$

14 Department of Animal Sciences, Chungbuk National University, Gaesin-dong, Cheongju,

15 Chungbuk 28864, Republic of Korea

16 E-mail: $\underline{\text { nhkim@,chungbuk.ac.kr }}$

17

18 Inchul Choi ${ }^{2}$

19 Department of Animal and Dairy Sciences, College of Agriculture and Life Sciences, Chungnam

20 National University, Daejeon, 34134, Republic of Korea.

21 E-mail: icchoi@,cnu.ac.kr 


\section{ABSTRACT}

The Rho-associated coiled-coil-containing protein serine/threonine kinases 1 and 2 (ROCK1 and ROCK2) are Rho subfamily GTPase downstream effectors that regulate cell migration, intercellular adhesion, cell polarity, and cell proliferation by stimulating actin cytoskeleton reorganization. Inhibition of ROCK proteins affects specification of the trophectoderm (TE) and inner cell mass (ICM) lineages, compaction, and blastocyst cavitation. However, the molecules involved in blastocyst formation are not known. Here, we examined developmental competence and levels of adherens/tight junction (AJ/TJ) constituent proteins, such as CXADR, OCLN, TJP1, and $\mathrm{CDH} 1$, as well as expression of their respective mRNAs, after treating porcine parthenogenetic four-cell embryos with Y-27632, a specific inhibitor of ROCK, at concentrations of $0,10,20,100 \mu \mathrm{M}$ for $24 \mathrm{~h}$. Following this treatment, the blastocyst development rates were $39.1,20.7,10.0$, and $0 \%$, respectively. In embryos treated with $20 \mu \mathrm{M}$ treatment, expression levels of CXADR, OCLN, TJP1, and CDH1 mRNA and protein molecules were significantly reduced $(P<0.05)$. FITC-dextran uptake assay revealed that the treatment caused an increase in TE TJ permeability. Interestingly, the majority of the four-cell and morula embryos treated with $20 \mu \mathrm{M}$ Y-27643 for $24 \mathrm{~h}$ showed defective compaction and cavitation. Taken together, our results indicate that ROCK activity may differentially affect assembly of $\mathrm{AJ} / \mathrm{TJ}$ s as well as regulate expression of genes encoding junctional proteins. 


\section{INTRODUCTION}

The Rho-associated coiled-coil-containing protein serine/threonine kinases (ROCK, referring collectively to both ROCK1 and ROCK2 isoforms) are downstream effectors of the Rho subfamily GTPase. They regulate cell migration, intercellular adhesion, cell polarity cytokinesis, and cell proliferation by affecting actin cytoskeleton reorganization and microtubule dynamic (Etienne-Manneville \& Hall 2002; Olson \& Sahai 2008; Ridley 1999). The biological roles and expression patterns of ROCK in mammalian embryo development have been intensively studied by pharmacological disruption of ROCK activity by the specific ROCK inhibitor Y-27632 and by using Real-Time qRT-PCR (Quantitative Reverse Transcription Polymerase Chain Reaction) and immunocytochemistry (ICC) approaches to visualize expression of ROCK genes and proteins (Duan et al. 2014; Kawagishi et al. 2004; Zhang et al. 2014). These studies demonstrated that early embryo developmental competence was affected in a dose/stagedependent manner when mouse and porcine embryos were exposed to Y-27632 (100 $\mu \mathrm{M}$ in mouse, 5, 10, and $15 \mu \mathrm{M}$ in porcine). For example, both ROCK1 and ROCK2 were detected during porcine preimplantation embryo development, but ROCK2 was more abundantly expressed in the trophectoderm (TE) of the blastocyst embryos compared to the expression levels of ROCK1. The majority of early cleavage stage embryos failed to progress to the blastocyst stage and remained arrested at the compaction/morula stages after they were exposed to Y-27632 at concentrations higher than $10 \mu \mathrm{M}$. Furthermore, porcine and mouse morula embryos treated with progressively higher concentrations of Y-27632 did not develop to the blastocyst stage either.

Recently, it has been reported that pharmacological inhibition of ROCK and RHO by Y-27632 and RHO inhibitor I from the two-cell stage enhanced the inner cell mass (ICM) lineage and suppressed TE lineage establishment through activation of Hippo signaling and disruption of cellular polarity due to suppression of the activity of the LATS kinase in the outer blastomere cells (Kono et al. 2014). Furthermore, treatment of blastocysts with Y-27632 disrupted ICM morphology and caused a transient reduction in the blastocyst cavity size and fetal loss after implantation (Laeno et al. 2013). In line with these findings, inhibition of ROCK activity by Y27632 induced defects of position-dependent HIPPO signaling and downregulation of $C d x 2$ expression in the emerging TE lineage (Cao et al. 2015). 
72 Taken together, these previous studies provided strong evidence that ROCK activity is involved 73 in blastocyst formation by regulating TE and ICM establishment via position-dependent HIPPO 74 signaling. However, it is not known why formation of the fluid-filled cavity is inhibited in early 75 cleaving embryos exposed to the ROCK inhibitor and why the size of the blastocyst cavity 76 transiently decreased in blastocysts treated with Y-27632. It is well documented that blastocyst formation requires water channels as physiological mediators of fluid movement across the TE, a correct $\mathrm{Na}^{+} / \mathrm{K}^{+-}$ATPase-generated trans-TE ion gradient for water accumulation, and a proper assembly of tight junction (TJ) proteins (Watson \& Barcroft 2001; Watson et al. 2004). Recently, ROCK has been reported to be down-regulated in TFAP2C-depleted embryos that were arrested in the transition between morula and blastocyst stages. These embryos exhibited defects of paracellular sealing because of the TJ disruption and had a phenotype resembling that of embryos treated with Y-27632 (Cao et al. 2015; Choi et al. 2012). Thus, we hypothesized that inhibition of ROCK activity leads to the impairment of TJ functions. Particularly, our recent finding that CXADR is required for adherens junction (AJ) and TJ assembly during porcine blastocyst formation (Kwon et al. 2016) also supports this hypothesis, because CXADR was reported to be directly associated with ROCK in human tumor cells (Saito et al. 2014). Here, we report that ROCK activity is involved in functional $\mathrm{TJ}$ assembly and paracellular sealing during the preimplantation development of parthenogenetic porcine embryos.

\section{MATERIALS AND METHODS}

All reagents were purchased from Sigma-Aldrich (St. Louis, MO, USA) unless stated otherwise.

\section{Collection of porcine oocytes and embryo culture}

Porcine-oocyte collection and embryo culture were carried out as described previously (Lee et al. 2015). Briefly, ovaries obtained from a local slaughter house were transported to the laboratory in Dulbecco's phosphate-buffered saline (DPBS) at $37^{\circ} \mathrm{C}$. Cumulus-oocyte complexes (COCs) aspirated from 3-6mm follicles were washed three times with 4-(2-hydroxyethyl)-1- 
100 piperazineethanesulfonic acid (HEPES)-buffered Tyrode's medium containing $0.1 \%(\mathrm{w} / \mathrm{v})$ 101 polyvinyl alcohol (HEPES-TL-PVA). Groups of $\sim 50$ COCs were incubated in the in vitro 102 maturation medium for $44 \mathrm{~h}$ at $39{ }^{\circ} \mathrm{C}$ and then denuded by pipetting in HEPES-TL containing 1 $103 \mathrm{mg} / \mathrm{mL}$ hyaluronidase for $2-3 \mathrm{~min}$. Denuded oocytes were treated with $50 \mu \mathrm{M}$ calcium ionophore 104 use at $39^{\circ} \mathrm{C}$ in a humidified atmosphere containing $5 \%$ of $\mathrm{CO}_{2}$.

\section{Quantification of transcript levels}

110

111

112

113

114

115

116

117

118

Isolation of mRNA from porcine blastocysts (ten samplings per each biological replicate) was carried out by using the Dynabeads mRNA Direct Kit (Dynal ASA, Oslo, Norway). First-strand cDNA was synthesized with the Superscript Reverse Transcriptase Enzyme (Invitrogen, Grand Island, NY, USA). Quantitative real-time RT-PCR (qRT-PCR) was carried out using cDNA synthesized with the Superscript Reverse Transcriptase Enzyme (Invitrogen, Grand Island, NY, USA) on a DNA Engine Opticon 2 Fluorescence Detection System (MJ Research, Waltham, MA, USA) with the DyNAmo SYBR Green qPCR Kit (Finnzymes Oy, Espoo, Finland). Transcripts of porcine CXADR, OCLN, and TJPI genes were amplified using specific primer pairs and conditions (Kwon et al., 2016). Relative quantification of gene expression was performed by the $2^{-\triangle \Delta C t}$ method from three technical and biological replicates for the control and ROCK inhibitortreated groups. GAPDH was used as the internal control in all experiments.

\section{Immunocytochemistry (ICC)}

The procedure was carried out as described previously (Lee et al., 2015). Porcine preimplantation embryos were washed in DPBS that contained polyvinyl alcohol $(1 \mathrm{mg} / \mathrm{mL})$ and fixed for $20 \mathrm{~min}$ in $3.7 \%(\mathrm{w} / \mathrm{v})$ paraformaldehyde dissolved in DPBS. Thereafter, embryos were permeabilized and blocked in DPBS that contained 0.5\% (v/v) Triton X-100 and 5\% donkey serum at room temperature for $1 \mathrm{~h}$. Embryos were then incubated with a rabbit polyclonal anti- 
128 CXADR antibody (Sigma), a mouse monoclonal anti-TJP1 antibody (ZO-1; Zymed, San 129 Francisco, CA, USA), or a mouse monoclonal anti-OCLN antibody (Zymed) in the blocking 130 solution (DPBS with 5\% donkey serum) overnight at $4{ }^{\circ} \mathrm{C}$, followed by incubation with Alexa 131 Fluor 488- and 594-conjugated antibodies (Molecular Probes, Eugene, OR, USA) as secondary 132 antibodies. The embryos were then mounted in Vectashield containing 4',6-diamidino-2133 phenylindole (DAPI; Vector Laboratories, Burlingame, CA, USA) and analyzed by a laser134 scanning confocal system with a krypton-argon ion laser under a Leica DM IRB inverted 135 microscope (DM IRB; Leica, Wetzlar, Germany).

\section{Treatment of embryos with the ROCK inhibitor Y-27632}

138 To examine the effects of the ROCK inhibitor Y-27632 on embryo development and polarized distribution of AJ and TJ proteins, late four-cell and morula embryos were incubated in $30 \mu 1$ droplets supplemented with Y-27632 at final concentrations of 10, 20, and $100 \mu \mathrm{M}$ for $24 \mathrm{~h}$ at $39^{\circ} \mathrm{C}$ in a humidified atmosphere containing $5 \%$ of $\mathrm{CO}_{2}$. Since the stock solution of Y-27632 was prepared in dimethyl sulfoxide (DMSO), control embryos were treated with the equivalent amount of DMSO and cultured until use.

\section{TJ permeability assay using FITC-dextran uptake}

To assess the effects of inhibition of ROCK activity on TJ permeability, DMSO vehicle control embryos and blastocysts derived from morula embryos treated with $10 \mu \mathrm{M}$ and $20 \mu \mathrm{M}$ of Y-27632 were incubated in the culture medium containing $1 \mathrm{mg} / \mathrm{mL}$ 40-kDa (fluorescein isothiocyanate) FITC-dextran for $30 \mathrm{~min}$ at $37^{\circ} \mathrm{C}$. Then, they were immediately washed several times in 50- $\mu$ l droplets of PZM5 to remove FITC-dextran on the surface of embryos, placed into a clean droplet of PZM5, and visualized under an inverted fluorescence microscope (TE2000U;

152 Nikon, Tokyo, Japan).

\section{Statistical analysis}


155 were subjected to the analysis of variance (ANOVA), whereas data from the tight junction 156 permeability assay were analyzed by the chi-square test using the Statistical Analysis System 157 software (Statistical Analysis System, Inc., Cary, NC, USA). The data are presented as the mean $158 \pm$ standard error of the mean (SEM). Differences with $P$ values $<0.05$ were considered 159 statistically significant unless stated otherwise.

\section{RESULTS}

162 163

164

165

166

167

168

169

170

171

172

173

174

175

176

177

178

179

180

181

182

Blastocyst development of porcine embryos treated with the specific ROCK inhibitor Y27632

To evaluate the effects of ROCK inhibition on blastocyst development, we treated the four-cell embryos with 0 (control), 10, 20, and $100 \mu \mathrm{M}$ Y-27632 for $24 \mathrm{~h}$. We observed that exposure to the inhibitor led to significantly lower blastocyst development rates $(39.1,20.7,10.0$, and $0 \%$, respectively) (Fig. 1A). The effects were significantly greater at higher Y-27632 concentrations (20 and $100 \mu \mathrm{M}$ ). The embryos that failed to develop to the blastocyst stage were arrested at the pre-compaction stage in presence of 10 and $20 \mu \mathrm{M}$ Y-27632 (Figs. 1B and 2A).

Next, we treated the four-cell and morula embryos with $20 \mu \mathrm{M}$ Y-27632 for $24 \mathrm{~h}$ and examined the stage-dependent effects of the ROCK inhibitor on morphological responses such as compaction and cavitation at 120 h.p.a (hours post activation). The majority of the four-cell embryos did not develop beyond the compacted/morula stage and remained at the four-cell stage as a result of treatment (Fig. 2A, B). Furthermore, while $49.1 \%$ of control morula reached the blastocyst stage with cavity and expansion, only $9.2 \%$ of morula treated with the ROCK inhibitor developed to the blastocyst (Figure 2B). At 120 h.p.a, we found that the total cell number in embryos treated with $20 \mu \mathrm{M}$ Y-27632 was lower than that in control embryos $(16.25 \pm 2.1$ and $29.7 \pm 4.4$, respectively) (Fig. 2C)

We also investigated the subcellular localization of the CXADR protein, which has been reported to be associated with ROCK activity, to determine whether ROCK affects polarized distribution of adhesion and tight junction proteins. In embryos arrested at the precompaction stage by their exposure to $100 \mu \mathrm{M}$ Y-27632 for $24 \mathrm{~h}$, the fluorescent signals were dispersed in 
183 the cytoplasm and very weak signals were detected at $48 \mathrm{~h}$ after the treatment, but control 184 embryos developed to the morula stage and showed continuous thin lines at the apical region of

185

186

187

188

189

190

191

192

193

194

195

196

197

198

199

200

201

202

203

204

205

206

207

208

209

210

211 cells (Fig. 2D). Embryos treated with the inhibitor at the morula stage for 24 h showed relatively visible continuous lines along the cell-cell boundaries compared to those in the four-cell treatment group but less distinct than those in control blastocysts at 120 h.p.a (Fig. 2D). These results suggested that ROCK activity affects cellular polarization of adhesion and tight junction proteins.

\section{Inhibition of AJ and TJ assembly in embryos by Y-27632}

Based on our findings and previous reports regarding the role of ROCK in blastocyst formation (Choi et al. 2012; Kawagishi et al. 2004; Zhang et al. 2014), we speculated that inhibition of the cavitation onset in Y-27632-treated embryos is caused by mislocalization or AJ and TJ proteins and impaired TJ assembly. Thus, we examined localization and expression levels of CXADR, OCLN, TJP1, and CDH1 mRNA and protein molecules, which are essential for the integrity of the AJ-TJ complex, by using ICC and qRT-PCR at the blastocyst stage. In embryos exposed to $20 \mu \mathrm{M}$ Y-27632, we observed that the CXADR and TJP1 proteins were barely detected at the apical regions of the cell-cell boundaries. In addition, while the OCLN protein was expressed in continuous lines at the apical edge of cell-cell contacts, its total expression in the treated blastocyst was very weak (Fig. 3A). The relative levels of CXADR, OCLN, TJP1, and CDH1 mRNA transcripts were also significantly lower in embryos from the Y-27632 treatment group $(P<0.05$, Fig. 3B).

These findings led us to hypothesize that integrity of the TJ complex was disrupted by mislocalization and lower expression levels of tight junction proteins in presence of the ROCK inhibitor. To examine the functional barrier properties of the tight junction assembly, we treated morula embryos with $10 \mu \mathrm{M}$ and $20 \mu \mathrm{M}$ Y -27632 for $24 \mathrm{~h}$ and cultured them until the blastocyst stage. At 144 h.p.a, we exposed blastocysts, including control ones treated with DMSO, to 40kDa FITC-dextran. We found a significant difference in the tight junction permeability in blastocysts from control and inhibitor-treated groups $(P<0.05)$, but no difference between the two treatment groups (Table 1). This observation demonstrated that inhibition of ROCK activity 
212 led to impairment of tight junction assembly during porcine blastocyst cavitation.

213

\section{DISCUSSION}

215 Previous studies reported that inhibition of ROCK activity by its specific inhibitor Y-27632 216 prevented blastocyst cavitation, tight junction biogenesis, TE and ICM formation, establishment of the intracellular polarity in the form of the apical-basal axis, and affected developmental competency in a dose- or a stage-dependent manner in mouse (Cao et al. 2015; Duan et al. 2014; Kawagishi et al. 2004; Kono et al. 2014). The defects found in embryos treated with the ROCK inhibitor were attributed to the loss of position-dependent HIPPO signaling that is mediated via the RHO-ROCK signaling(Kono et al. 2014). A number of studies have shown that ion gradient, adherens junctions, tight junctions, and water channels are properly expressed and localized on the outer cells of embryos and the TE to form blastocyst (reviewed in (Choi et al. 2012; Watson \& Barcroft 2001; Watson et al. 2004)). However, the specific cellular events and molecules involved in developmental perturbations in early embryos induced by ROCK inhibition were not defined. Here, focusing on tight junction assembly, we investigated how inhibition of ROCK activity affected preimplantation development of porcine embryos.

First, we demonstrated that treatment with Y-27632 resulted in the failure of early porcine embryo development in a dosage-dependent manner. In line with previous results obtained in the

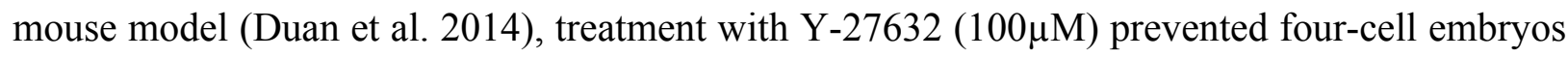
from undergoing compaction and also inhibited the morula-to-blastocyst transformation during porcine preimplantation development (summarized in Fig. 4A). A possible explanation of defective compaction and impaired blastocyst formation is that the two ROCK isoforms, ROCK1 and ROCK2, have different stage-specific expression patterns and subcellular localizations during preimplantation development and, therefore, can have different biological activities (Zhang et al. 2014). ROCK1 is predominantly detected in the cytoplasm and localized mainly at the cell-cell boundaries at the four-cell to morula stages. However, ROCK1 is barely detected in the blastocyst, while ROCK 2 is present in both the cytoplasm and the nucleus with predominant expression in the TE nuclei (Zhang et al. 2014) (see schematic representation of expression and localization of ROCK1 and ROCK2 in porcine embryos in Fig. 4). Considering the spatial and temporal expression patterns of ROCK1 and ROCK2 and the stage-dependent impairment of 
242 embryo development by ROCK inhibition, we suggest that the arrest of embryos at pre243 compaction is associated with inhibition of ROCK1 rather than ROCK2, because ROCK1 is 244 involved in cell adhesion via regulating actomyosin contraction and association with E-cadherin 245 complexes (Shi et al. 2013; Smith et al. 2012).

Next, we examined subcellular localization of CXADR, which was recently reported to be required for adherens and tight junction formation during porcine blastocyst development (Kwon et al. 2016) and shown to directly associated with ROCK1 and ROCK2 in human carcinoma cells (Saito et al. 2014). The observed disruption of localization of the CXADR protein and reduced expression of the $C X A D R$ gene indicated that clustering of junctional proteins complex and its functional barrier can be mediated by CXAD.R. Thus, we examined single-pass membrane protein (CXADR), four-pass membrane domain (OCLN), and cytoplasmic plaque protein (TJP1), and adherens protein $(\mathrm{CDH} 1)$, found a significant decrease in the protein and mRNA expression levels, and demonstrated increased paracelluar permeability in the ROCK inhibitor treated blastocysts (Table 1). The deficient barrier function in blastocysts was likely caused by the disruption of tight junction assembly and deregulation of gene expression on the morula-to-blastocyst developmental stage. A growing body of evidence has demonstrated that adherens and tight junctions are both linked intracellularly to F-actin via adaptor proteins such as TJP1 and OCLN, which are phosphorylated by ROCK (Aijaz et al. 2006; Balda et al. 1996; Gopalakrishnan et al. 1998; Hartsock \& Nelson 2008; Hirase et al. 2001; Ohnishi et al. 2004; Sahai \& Marshall 2002; Wittchen et al. 1999; Yamamoto et al. 2008). In addition, nuclear ROCK 2 has been reported to be involved in transcriptional regulation of the trophoblast lineage and co-localized with the p300 acetyltransferase (Carey et al. 2013; Rodriguez et al. 2004; Tanaka et al. 2006). Converging lines of evidence suggest that inhibition of cytoplasmic ROCK1 activity in morula can adversely affect the interaction of cytoskeleton F-actin with TJP1 and phosphorylation of the carboxy-terminal domain of OCLN, which is important for paracellular sealing of the TE during the transition stage (Fig. 4B). Furthermore, inhibition of nuclear ROCK2 activity may lead to reduced expression of the CXADR, OCLN, TJP, and CDH1 genes via transcriptional regulation associated with p300 activity (Fig. 4B). developmental steps such as compaction and cavitation. We suggest that observed defective 
272 embryo development and lower gene expression of $\mathrm{AJ} / \mathrm{TJ}$ associated genes in presence of the 273 specific ROCK inhibitor are caused by specific temporo-spatial inhibition of distinct ROCK1 274 and ROCK2 functions in porcine embryos. In this context, it will be important to establish the 275 molecular mechanisms of differential subcellular targeting of ROCK isoforms during 276 intracellular polarization and adherens/tight junction biogenesis throughout early embryo 277 development.

278

279 
280

281

282

283

284

285

286

287

288

289

290

291

292

293

294

295

296

297

298

299

300

301

302

303

304

305

306

307

308

309

310

311

312

313

314

315

\section{REFERENCES}

Aijaz S, Balda MS, and Matter K. 2006. Tight junctions: Molecular architecture and function. In: Jeon KW, ed. International Review of Cytology - a Survey of Cell Biology, Vol 248. San Diego: Elsevier Academic Press Inc, 261-298.

Balda MS, Whitney JA, Flores C, González S, Cereijido M, and Matter K. 1996. Functional dissociation of paracellular permeability and transepithelial electrical resistance and disruption of the apical-basolateral intramembrane diffusion barrier by expression of a mutant tight junction membrane protein. The Journal of Cell Biology 134:1031-1049. 10.1083/jcb.134.4.1031. doi: 10.1083/jcb.134.4.1031

Cao Z, Carey TS, Ganguly A, Wilson CA, Paul S, and Knott JG. 2015. Transcription factor AP-2Y induces early $\mathrm{Cdx2}$ expression and represses HIPPO signaling to specify the trophectoderm lineage. Development 142:1606-1615. 10.1242/dev.120238. doi: 10.1242/dev.120238

Carey TS, Choi I, Wilson CA, Floer M, and Knott JG. 2013. Transcriptional Reprogramming and Chromatin Remodeling Accompanies Oct4 and Nanog Silencing in Mouse Trophoblast Lineage. Stem Cells and Development 23:219-229. 10.1089/scd.2013.0328. doi: $10.1089 /$ scd.2013.0328

Choi I, Carey TS, Wilson CA, and Knott JG. 2012. Transcription factor AP-2 $\gamma$ is a core regulator of tight junction biogenesis and cavity formation during mouse early embryogenesis. Development 139:4623-4632. 10.1242/dev.086645. doi: 10.1242/dev.086645

Duan X, Chen K-L, Zhang Y, Cui X-S, Kim N-H, and Sun S-C. 2014. ROCK inhibition prevents early mouse embryo development. Histochemistry and Cell Biology 142:227-233. 10.1007/s00418014-1201-6. doi: 10.1007/s00418-014-1201-6

Etienne-Manneville S, and Hall A. 2002. Rho GTPases in cell biology. Nature 420:629-635.

Gopalakrishnan S, Raman N, Atkinson SJ, and Marrs JA. 1998. Rho GTPase signaling regulates tight junction assembly and protects tight junctions during ATP depletion. American Journal of Physiology - Cell Physiology 275:C798-C809.

Hartsock A, and Nelson WJ. 2008. Adherens and tight junctions: Structure, function and connections to the actin cytoskeleton. Biochimica Et Biophysica Acta-Biomembranes 1778:660-669. 10.1016/j.bbamem.2007.07.012. doi: 10.1016/j.bbamem.2007.07.012

Hirase T, Kawashima S, Wong EYM, Ueyama T, Rikitake Y, Tsukita S, Yokoyama M, and Staddon JM. 2001. Regulation of Tight Junction Permeability and Occludin Phosphorylation by RhoAp160ROCK-dependent and -independent Mechanisms. Journal of Biological Chemistry 276:10423-10431. 10.1074/jbc.M007136200. doi: 10.1074/jbc.M007136200

Kawagishi R, Tahara M, Sawada K, Ikebuchi Y, Morishige K, Sakata M, Tasaka K, and Murata Y. 2004. Rho-kinase is involved in mouse blastocyst cavity formation. Biochemical and Biophysical Research Communications 319:643-648. doi: http://dx.doi.org/10.1016/j.bbrc.2004.05.040 
316

317

318

319

320

321

322

323

324

325

326

327

328

329

330

331

332

333

334

335

336

337

338

339

340

341

342

343

344

345

346

347

348

349

350

351

352

Kono K, Tamashiro DAA, and Alarcon VB. 2014. Inhibition of RHO-ROCK signaling enhances ICM and suppresses TE characteristics through activation of Hippo signaling in the mouse blastocyst. Developmental Biology 394:142-155. doi: http://dx.doi.org/10.1016/j.ydbio.2014.06.023

Kwon J, Kim N-H, and Choi I. 2016. CXADR is required for AJ and TJ assembly during porcine blastocyst formation. Reproduction. doi: 10.1530/rep-15-0397

Laeno AMA, Tamashiro DAA, and Alarcon VB. 2013. Rho-Associated Kinase Activity Is Required for Proper Morphogenesis of the Inner Cell Mass in the Mouse Blastocyst. Biology of Reproduction 89:122, 121-113. doi: 10.1095/biolreprod.113.109470

Lee S-H, Kwon J-W, Choi I, and Kim N-H. 2015. Expression and function of transcription factor AP-2 $\gamma$ in early embryonic development of porcine parthenotes. Reproduction, Fertility and Development.-. doi: http://dx.doi.org/10.1071/RD14198

Ohnishi H, Nakahara T, Furuse K, Sasaki H, Tsukita S, and Furuse M. 2004. JACOP, a Novel Plaque Protein Localizing at the Apical Junctional Complex with Sequence Similarity to Cingulin. Journal of Biological Chemistry 279:46014-46022. doi: 10.1074/jbc.M402616200

Olson MF, and Sahai E. 2008. The actin cytoskeleton in cancer cell motility. Clinical \& Experimental Metastasis 26:273-287. doi: 10.1007/s10585-008-9174-2

Ridley AJ. 1999. Stress fibres take shape. Nat Cell Bio/ 1:E64-E66.

Rodriguez TA, Sparrow DB, Scott AN, Withington SL, Preis JI, Michalicek J, Clements M, Tsang TE, Shioda T, Beddington RSP, and Dunwoodie SL. 2004. Cited1 Is Required in Trophoblasts for Placental Development and for Embryo Growth and Survival. Molecular and Cellular Biology 24:228-244. doi: 10.1128/mcb.24.1.228-244.2004

Sahai E, and Marshall CJ. 2002. ROCK and Dia have opposing effects on adherens junctions downstream of Rho. Nature Cell Biology 4:408-415. doi: 10.1038/ucb796

Saito K, Sakaguchi M, Iioka H, Matsui M, Nakanishi H, Huh NH, and Kondo E. 2014. Coxsackie and adenovirus receptor is a critical regulator for the survival and growth of oral squamous carcinoma cells. Oncogene 33:1274-1286. doi: 10.1038/onc.2013.66

Shi J, Wu X, Surma M, Vemula S, Zhang L, Yang Y, Kapur R, and Wei L. 2013. Distinct roles for ROCK1 and ROCK2 in the regulation of cell detachment. Cell Death Dis 4:e483. doi: http://www.nature.com/cddis/journal/v4/n2/suppinfo/cddis201310s1.html

Smith AL, Dohn MR, Brown MV, and Reynolds AB. 2012. Association of Rho-associated protein kinase 1 with E-cadherin complexes is mediated by p120-catenin. Molecular Biology of the Cell 23:99-110. doi: 10.1091/mbc.E11-06-0497

Tanaka T, Nishimura D, Wu R-C, Amano M, Iso T, Kedes L, Nishida H, Kaibuchi K, and Hamamori Y. 2006. Nuclear Rho Kinase, ROCK2, Targets p300 Acetyltransferase. Journal of Biological Chemistry 281:15320-15329. doi: 10.1074/jbc.M510954200

Watson AJ, and Barcroft LC. 2001. Regulation of blastocyst formation. Frontiers in BioscienceLandmark 6:D708-D730. doi: 10.2741/Watson 
353

354

355

356

357

358

359

360

361

362

363

364

365

366

367

368

369

370

371

372

373

4

5

6

\section{7}

Watson AJ, Natale DR, and Barcroft LC. 2004. Molecular regulation of blastocyst formation. Animal Reproduction Science 82-83:583-592. doi: http://dx.doi.org/10.1016/j.anireprosci.2004.04.004

Wittchen ES, Haskins J, and Stevenson BR. 1999. Protein Interactions at the Tight Junction: ACTIN HAS MULTIPLE BINDING PARTNERS, AND ZO-1 FORMS INDEPENDENT COMPLEXES WITH ZO-2 AND ZO-3. Journal of Biological Chemistry 274:35179-35185. doi: 10.1074/jbc.274.49.35179

Yamamoto M, Ramirez SH, Sato S, Kiyota T, Cerny RL, Kaibuchi K, Persidsky Y, and Ikezu T. 2008. Phosphorylation of claudin-5 and occludin by Rho kinase in brain endothelial cells. American Journal of Pathology 172:521-533. doi: 10.2353/ajpath.2008.070076

Zhang JY, Dong HS, Oqani RK, Lin T, Kang JW, and Jin DI. 2014. Distinct roles of ROCK1 and ROCK2 during development of porcine preimplantation embryos. Reproduction 148:99-107. doi: 10.1530/rep-13-0556 


\section{1}

Blastocyst development of the four-cell porcine embryos treated with ROCK inhibitor, Y27632.

(A) The blastocyst development rates of embryos exposed to different concentrations of $Y$ 27632 (control 0, treatment 10, 20, and $100 \mu \mathrm{M}$ ) for 24 hours at the four-cell stage $(77,70$, 74, and 70 embryos, respectively; 22-27 embryos/ replicate). Error bar means \pm SEM, different letters are significantly different $(\mathrm{P}<0.05)$. n.d. not detected. (B) Representative images of embryos treated with the different concentrations at 48 h.p.a (hours post activation) and the embryos cultured until 144 h.p.a (at expanding stages) after 24 hours treatment of the four cell embryos with different concentrations of Y-27632. Bar $150 \mu \mathrm{M}$. 
A

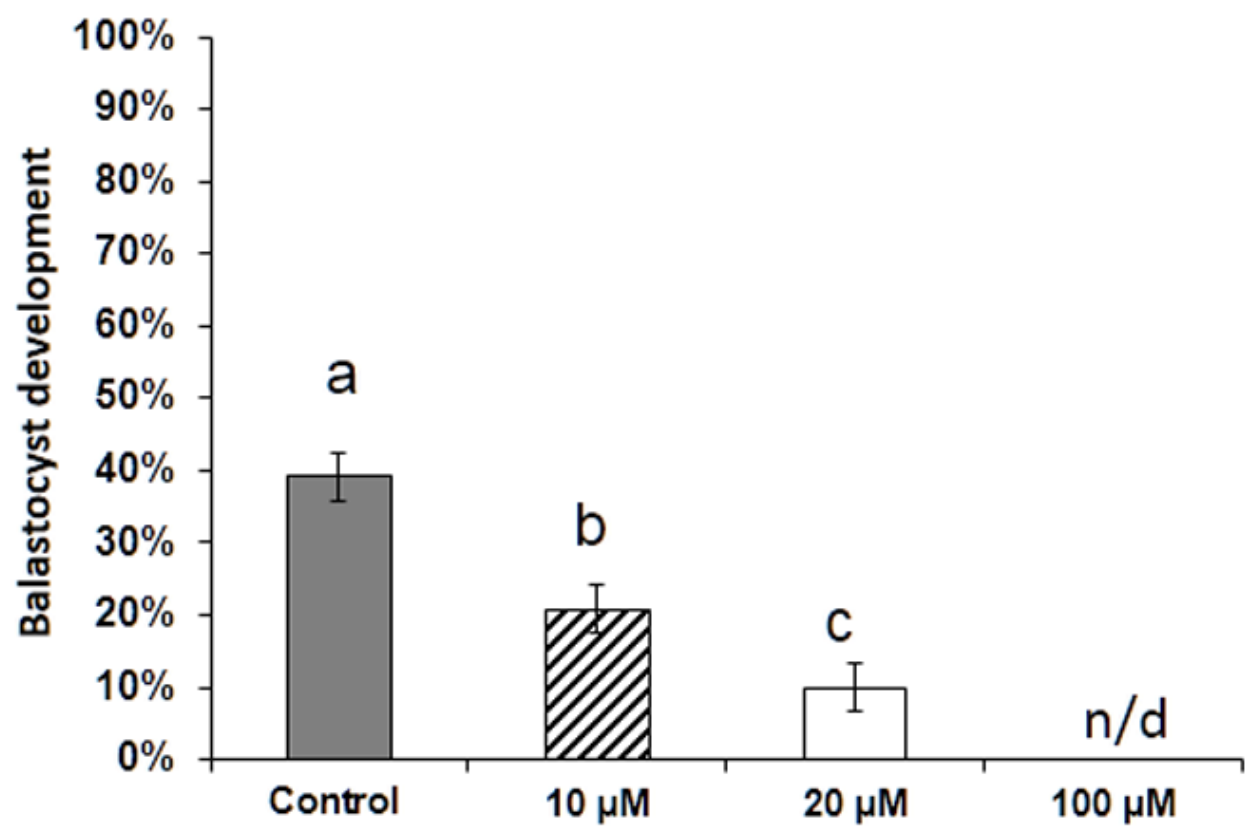

B
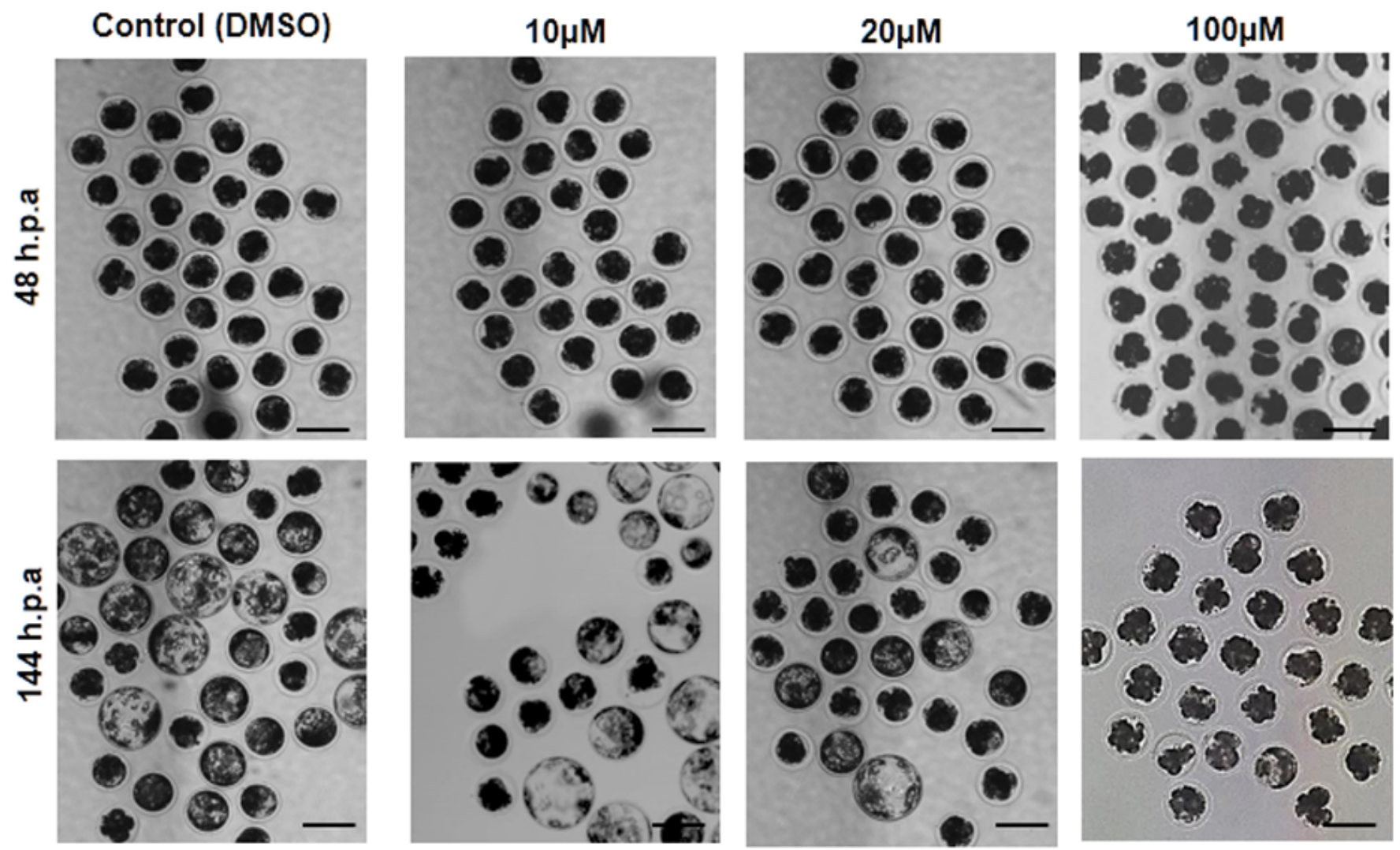


\section{2}

Stage specific effects of ROCK inhibitor on compaction and cavitation.

(A) The percentages of 92 and 71 embryos treated with $20 \mu \mathrm{M}$ Y-27632 at the four-cell or morula stages, respectively that developed to morula, and blastocyst at 96 and 120 h.p.a,. (B) Representative images of porcine embryos at 120 h.p.a. following treatment of the fourcell and morula, respectively, with $20 \mu \mathrm{M}$ Y-27632 for 24 (C) Total cell number of embryos at 120 h.p.a after treatment of morula embryos with $20 \mu \mathrm{M}$ Y-27632; For this analysis, blastomeres of 24 morula embryos were counted in the control and treatment group. Error bar means \pm SEM, Astrisks $(*)$ indicate means that are significantly different from the control $(P<0.05)$. (D) Localization of CXADR protein in porcine embryos. CXADR proteins were localized to cell to cell boundaries in the control compacted embryo and the blastocyst but in $20 \mu \mathrm{M}$ Y-27632 treated embryos was disused to cytoplasm or not distinct continuous lines on apical regions of each blastomeres. 
A

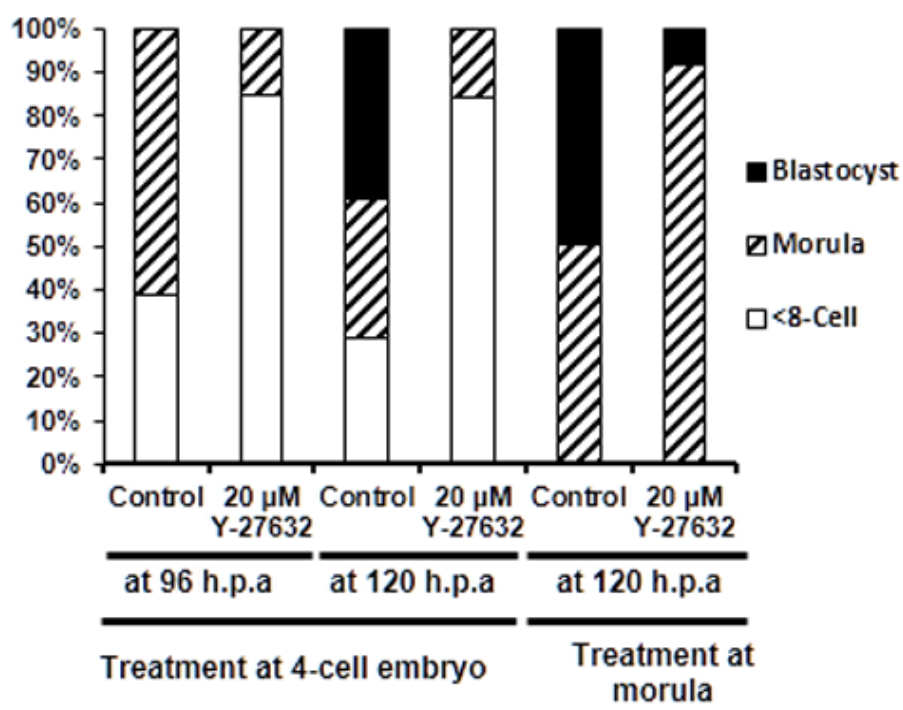

B

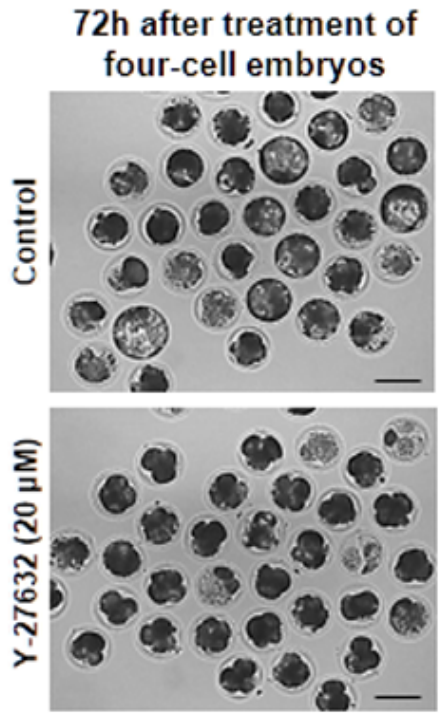

24h after treatment of Morula embryos
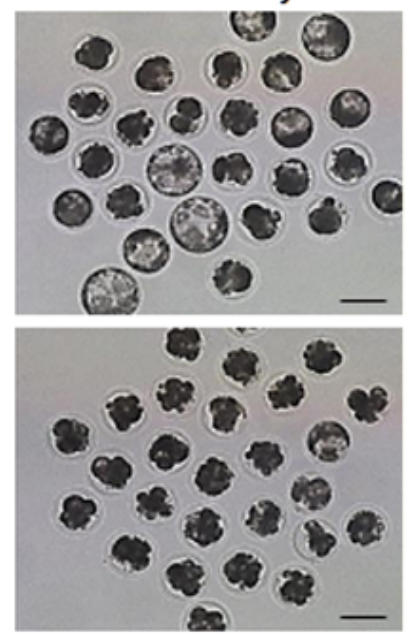

C

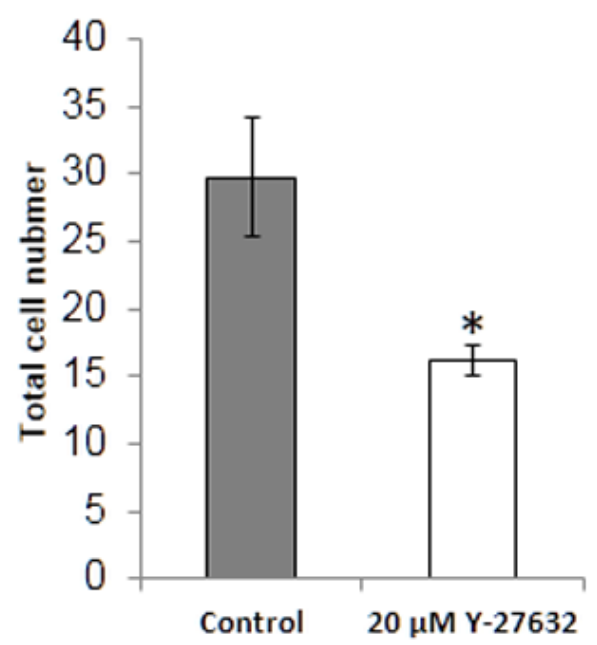

D

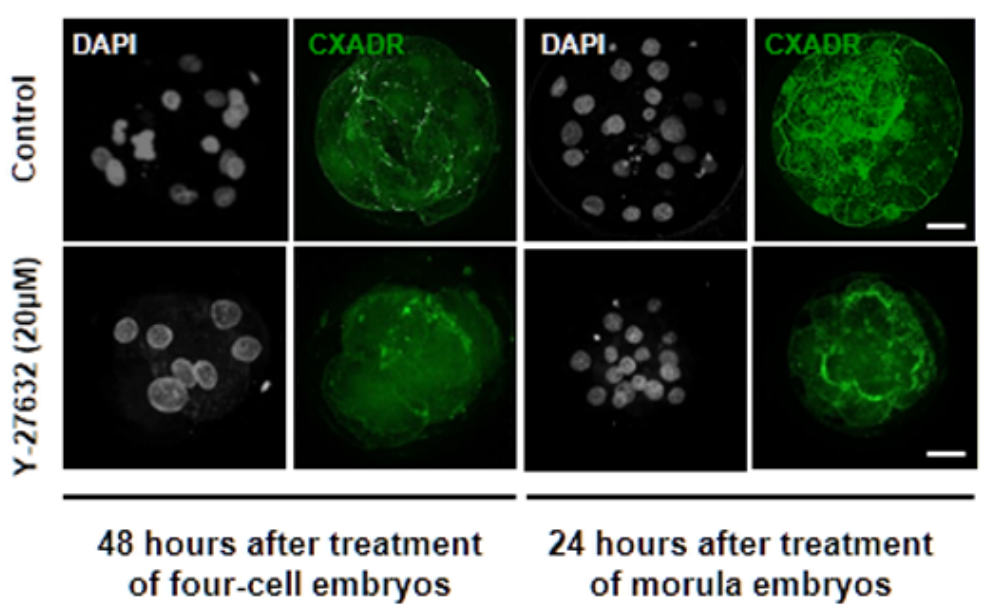




\section{3}

Expression of tight junction and adherens proteins.

(A) Localization of tight junction associated proteins (CXADR, OCLN, and TJP) and adherens

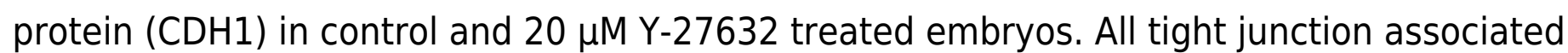
proteins and $\mathrm{CDH} 1$ were reduced in the treatment group. (B) Relative transcription levels of CXADR, OCLN TJP1, and CDH1 of embryos treated with $10 \mu \mathrm{M}$ Y-27632 to non-treated control blastocyst (ten per biological and three times technical replicates). Error bar means \pm SEM, Astrisks $(*)$ indicate means that are significantly different from the control $(P<0.05)$. RQ (Relative Quantification) 


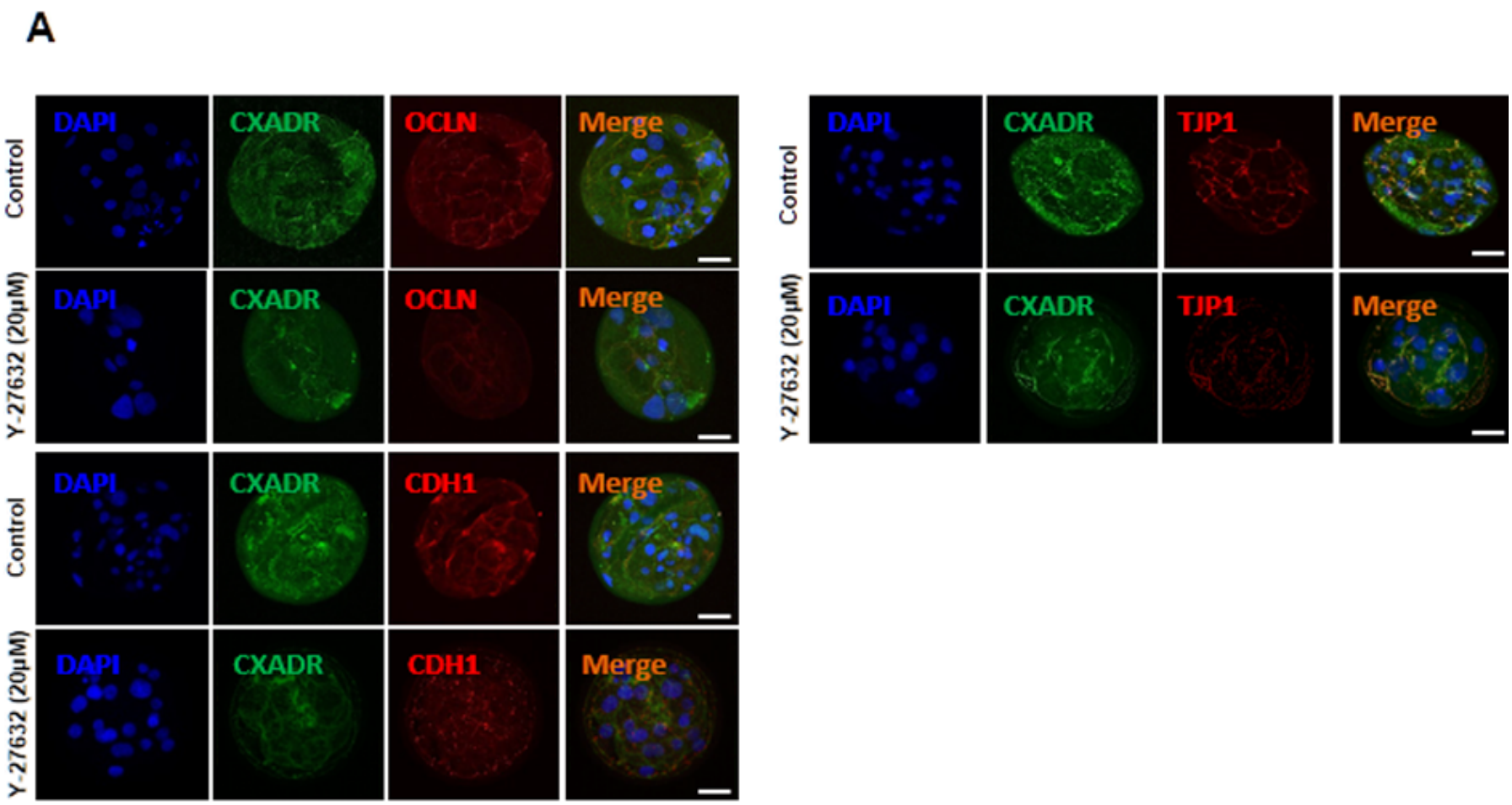

B

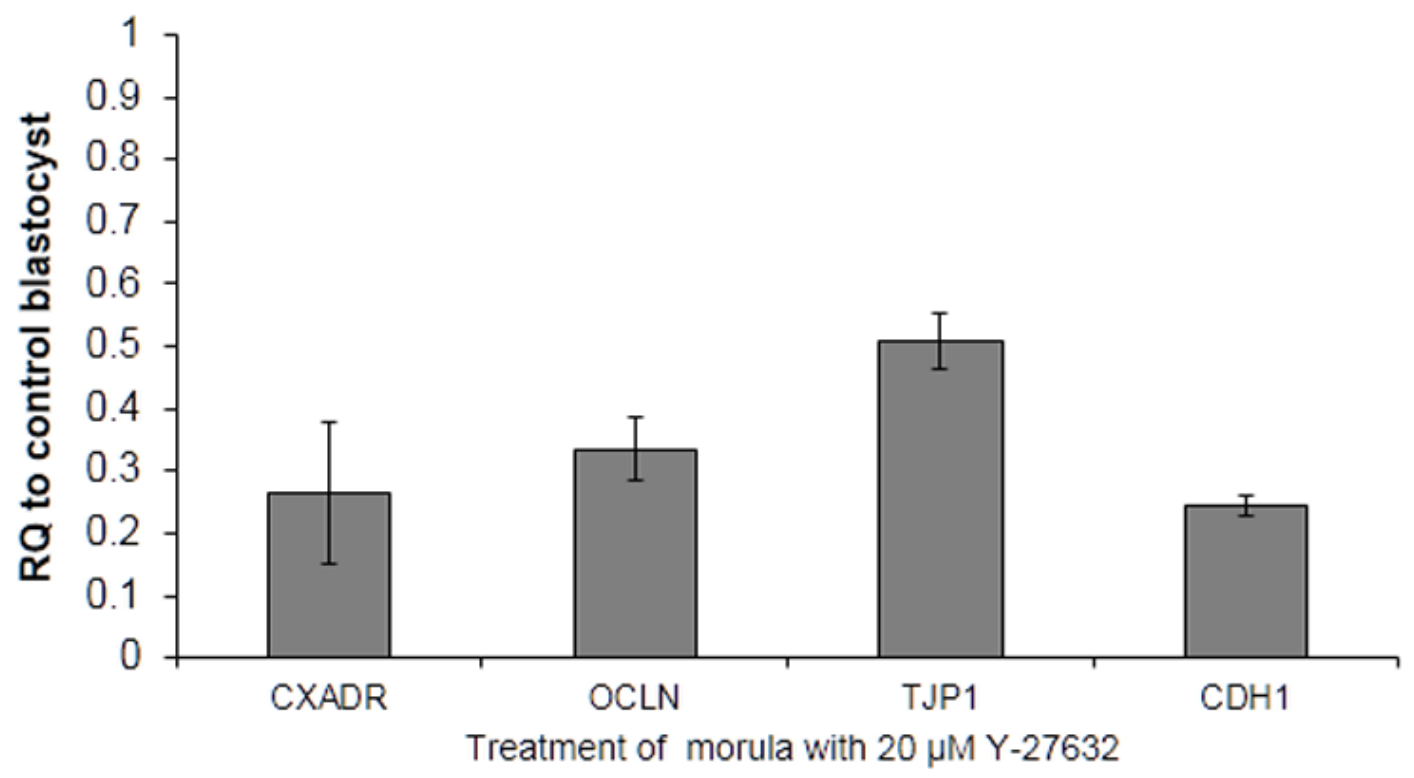




\section{4}

Summary of effects of ROCK inhibition on embryo development and model of regulation of ROCK activity in porcine embryos.

(A) Schematic view of embryo development and the results of ROCK inhibition experiments. Yellow and red (cell polarity); hatched bar (DMSO treatment); filled bar (Y-27632 treatment). Green (expression of ROCK1 or ROCK2); Circle (white: cell membrane; center: nucleus; others: cytoplasm), adapted from Zhang et al., 2014. (B) Model of ROCK activitiy in porcine embryos. Cytoplasmic ROCK1 is involved in phosphorylation of OCLN and linked to actin stability, providing establishment of tight junction, and paracellular sealing during morula to blastocyst transition. Tight junction also affects expression of genes related with tight junction including OCLN, TJP1, and CXADR via nuclear ROCK2 mediated by p300, histone acetyltransferase at the blastocyst to maintain junctional complexes. 
A

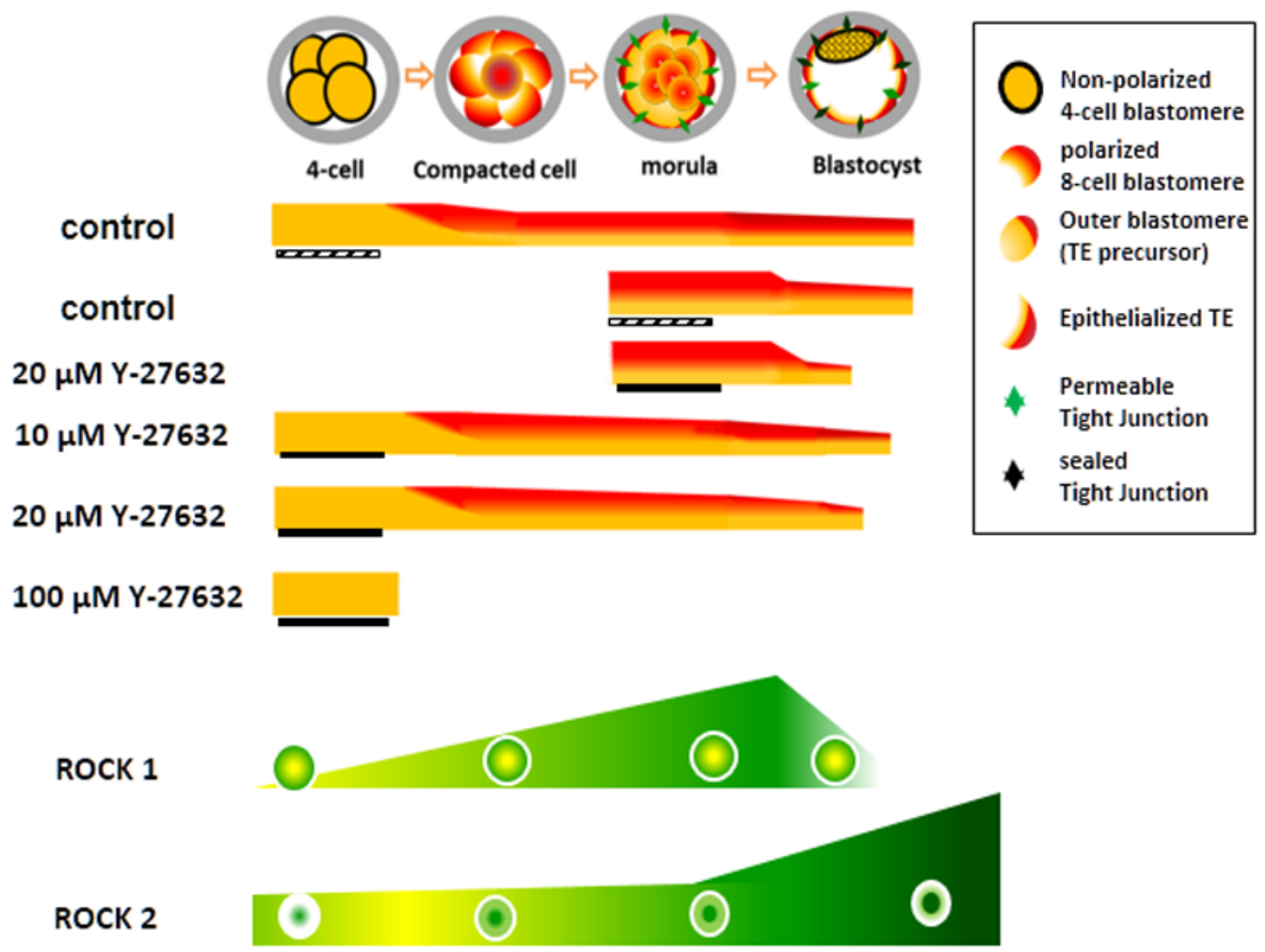

B

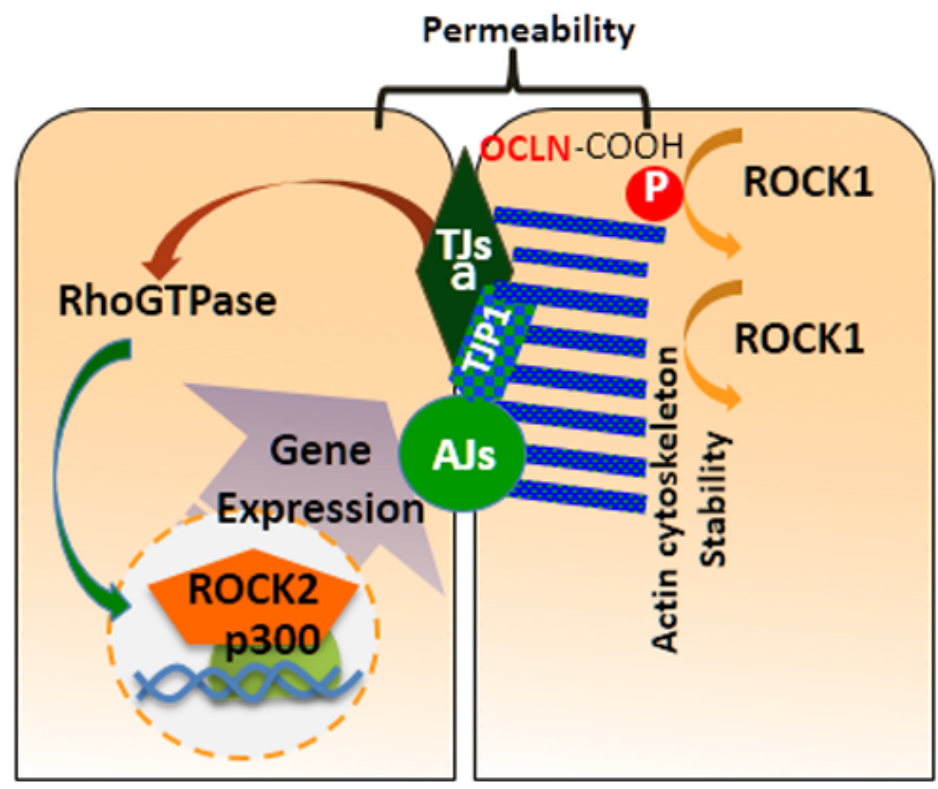




\section{Table $\mathbf{1}$ (on next page)}

FITC uptake assay after treatment of Y-27632 at the morula stage

FITC-Dextrans uptake assay for paracellular sealing in blastocyst. Y-27632 treated embryos showed that defects of paracellular sealing compared with control embryos. 
1 Table 1. FITC uptake assay

\begin{tabular}{lccc}
\hline & DMSO & $10 \mu \mathrm{M}$ & $20 \mu \mathrm{M}$ \\
\hline FITC dextran Positive & 3 & 7 & 10 \\
FITC dextran Negative & 59 & 25 & 21
\end{tabular}

2

Y-27632 treatment at the morula stage

3 